\title{
Hereditary haemochromatosis: a case of iron accumulation in the basal ganglia associated with a parkinsonian syndrome
}

\author{
J E Nielsen, L Neerup Jensen, K Krabbe
}

\begin{abstract}
Hereditary haemochromatosis is characterised by excessive parenchymal iron deposition, particularly in the liver. Usually hereditary haemochromatosis is not associated with neurological symptoms and iron deposition in the brain has not previously been described as a pathological phenomenon. A patient is reported with hereditary haemochromatosis and a syndrome of dementia, dysarthria, a slowly progressive gait disturbance, imbalance, muscle weakness, rigidity, bradykinesia, tremor, ataxia, and dyssynergia. The findings on MRI of a large signal decrease in the basal ganglia, consistent with excessive iron accumulation, indicate a causal relation to the symptoms. Although the neurological symptoms did not improve in our patient, hereditary haemochromatosis should be considered in the differential diagnosis of parkinsonian syndromes, because complications of iron induced organ injury may be prevented by phlebotomy.
\end{abstract}

(F Neurol Neurosurg Psychiatry 1995;59:318-321)

University Clinic of Neurology, Hvidovre Hospital, Copenhagen and Institute of Medical Biochemistry and Genetics, Laboratory of Medical Genetics, Section of Neurogenetics, University of Copenhagen, Denmark

J E Nielsen

Department of Pediatrics, Hillerød Hospital, Denmark L Neerup Jensen

Danish Research Center of Magnetic Resonance, Hvidovre Hospital, Denmark K Krabbe

Correspondence to: Dr J E Nielsen, Institute of Medical Genetics, Section of Neurogenetics, The Panum Institute, Buildin 24.4, Blegdamsvej 3, DK2200 Copenhagen $N$, Denmark.

Received 21 January 1995 and in revised form 8 May 1995

Accepted 11 May 1995 percentage saturation of transferrin and serum ferritin concentration, increased amounts of stainable iron in hepatocytes and an increased hepatic iron concentration. Most patients present with symptoms at the age of $40-50$, but some may present earlier, in the second or third decade. There is a sex difference and the frequency of male to female haemochromatosis ranges from $2: 1$ to $18: 1 .^{2}$ Neurological features associated with hereditary haemochromatosis are rare. Lethargy, psychomotor retardation, fatigue, confusion, hearing loss, and polyneuropathy have been described. ${ }^{3}$

To the best of our knowledge, there have been no reports of excessive iron deposition in the CNS in hereditary haemochromatosis, an otherwise well known pathological feature of the Hallervorden-Spatz syndrome and familial hypoceruloplasminaemia.

We report on a patient with hereditary haemochromatosis with a parkinsonian syndrome, most likely caused by excessive iron accumulation in the basal ganglia and cerebellum.

\section{Case report}

The patient, a 50 year old man with a normal psychomotor development, without known predisposition of any kind and no family history of hereditary haemochromatosis, had been admitted to a neurology department when 29 years old, because of a slowly progressive gait disturbance with episodes of unsteadiness and imbalance since the age of 26. He noticed muscular weakness, rigidity, and slowness of voluntary movement of the left arm and leg. His gait was described as reeling with a deviation towards the left, dragging his left foot. Tendon reflexes were normal, except for a very brisk right sided biceps reflex. Otherwise the neurological examination was normal. A pneumoencephalogram showed slight central atrophy of the cerebrum. Cervical pneumomyelography was normal, as were radiographs of the chest, spine, and skull. Blood tests, examination of CSF, and EEG were all reported to be within the normal range. He was discharged without any definite diagnosis.

Subsequently he was referred for a specialist opinion concerning his application for a disablement pension. For 18 years he had 
Figure 1 Coronal $(A)$ and transverse $(B, C)$

$M R I,(T 2$ weighted) of the brain, performed at high field strength of 1.5 Tesla with a double spin echo technique. There is a pronounced signal decrease in the caudate nucleus, the inner segment of the globus pallidus, the red nucleus, and substantia nigra, consistent with excessive iron accumulation. The dentate nucleus (not shown) was similar. There was no atrophy or focal abnormality seen.
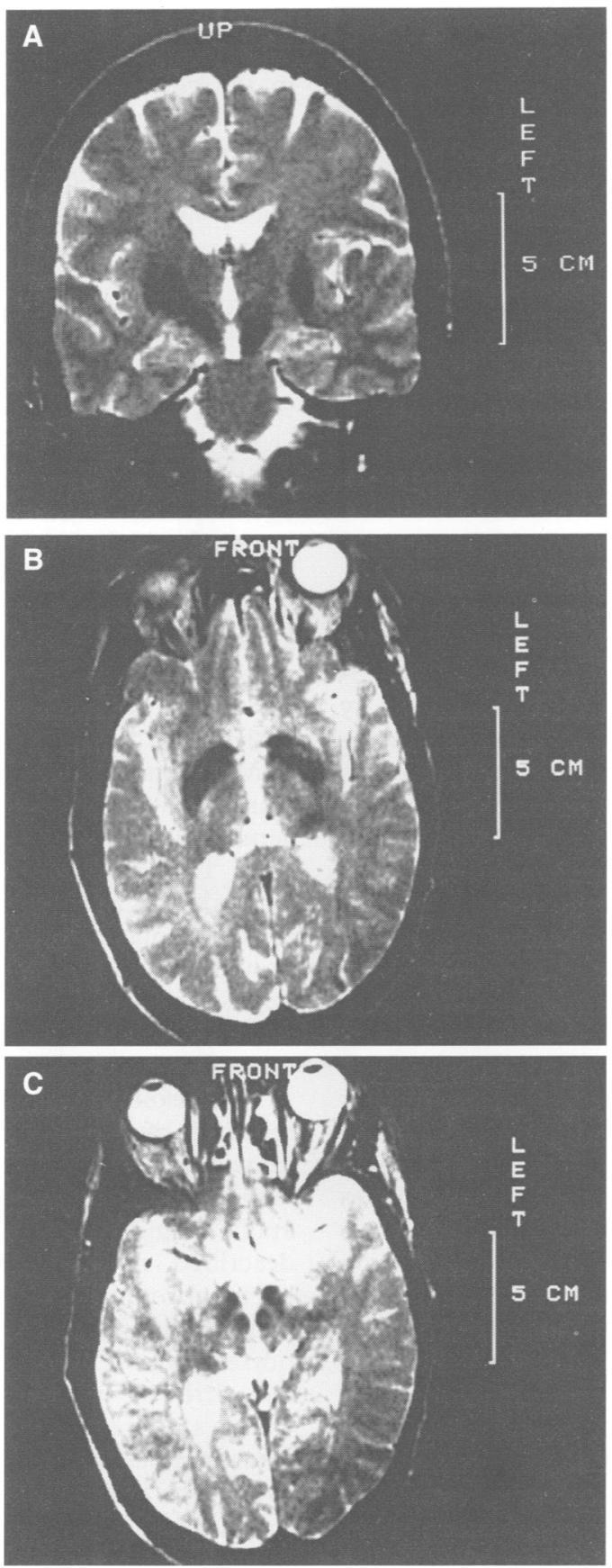

been working as an airport tractor driver. Four years ago he was transferred to an administrative job because of difficulties in motor function, a job he was now intellectually incapable of maintaining. The symptoms had been slowly progressive over the years, gradually also encompassing the right limbs, in such a way that using cutlery and dressing had become difficult. He also complained of fatigue, headache, a monotonous voice, and an impaired short-term memory. He had no cardiac complaints or arthralgiae, and no weight loss, abdominal pain, impotence, or episodes of alcohol misuse.

He was slightly impaired intellectually with a poor memory. His speech was monotonous, slow, and slurred with dysarthria and dysprosody, but with normal volume. His face was expressionless and mask-like and he blinked infrequently. A difference in the degree of pigmentation in the irides, the centres being brownish and hyperpigmented, was striking, but no Kayser-Fleischer ring was seen on slit lamp examination. Other cranial nerve functions were normal. Examination of the heart and the abdomen was normal, and there was no testicular atrophy.

A slight muscle atrophy was evident in the lower limbs, mainly distally on the left side. Muscular strength was moderately impaired in the upper limbs and in the lower limbs on the left in a pyramidal distribution. The muscular tone was increased in both upper and lower limbs in the form of rigidity, mainly on the left side, where there was also a discrete cogwheel phenomenon. Cutaneous sensation, joint position, and vibration senses were normal. Tendon reflexes were easily obtained in the upper limbs and were very brisk in the lower limbs, particularly on the left, with clonus at the ankle. The right plantar reflex was normal, whereas a Babinski's reflex was found on the left. Coordination was influenced by ataxia and severe dysdiadochokinesia. A combined static and postural tremor was noted, as well as truncal instability, with a tilting to the left. The gait was characterised by spasticity with circumduction of the left leg, holding the left arm in an adducted, flexed position; the step length and pace was normal. No involuntary movements were noted, but bradykinesia was seen. The skin was bronzed. The patient started treatment with levodopa. This resulted in an immediate improvement.

Normal findings included an ECG, blood cell counts, haemoglobin, erythrocyte sedimentation rate, serum concentrations of creatinine, carbamide, sodium, potassium, calcium, albumin, carbon dioxide, transaminase, alkaline phosphatase, total bilrubin, IgA, IgG, IgM, urate, a negative serum antinuclear antibody titre, and iron, plasma concentrations of fasting glucose and ceruloplasmin, and plasma prothrombin time. Urinary copper excretion was normal and the copper content in the liver $(5.2 \mathrm{mg} / \mathrm{kg})$ was also within the normal range $(5-15 \mathrm{mg} / \mathrm{kg}$ ). Serum ferritin (1313 pmol/1 (normal 33-666 $\mathrm{pmol} / \mathrm{l}$ ) and transferrin saturation (53\% (normal $<50 \%$ ) were high. Serum transferrin (25 $\mu \mathrm{mol} / 1$ (normal $31-57 \mu \mathrm{mol} / 1$ )) was low. A liver biopsy showed a heavy iron overload, but liver architecture was normal. Brain MRI (fig 1) showed a pronounced signal decrease in the caudate nucleus, the inner segment of the globus pallidus, the red nucleus, substantia nigra, and the dentate nucleus, consistent with excessive iron accumulation. No atrophy or focal abnormalities were seen. Liver MRI (fig 2) showed a pronounced signal intensity decrease, corresponding to a hepatic iron concentration of $70 \mu \mathrm{mol} / \mathrm{g}$ wet weight (normal range 1-9 $\mu \mathrm{mol} / \mathrm{g}$ ). HLA typing showed heterozygosity for the HLA system (HLAA2,3,B7,44).

Phlebotomy was started. After eight treatments the fatigue, the headache, and the skin bronzing had disappeared. There were no other improvements. 
Figure 2 T2 weighted $M R I$ of the liver with spin echo and gradient echo sequences, showed a pronounced signal intensity decrease. There are no focal abnormalities and the spleen is normal.

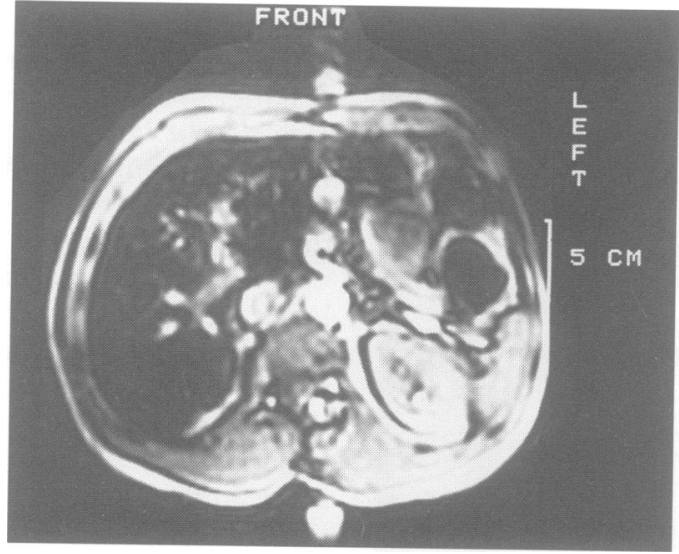

\section{Discussion}

The diagnosis of hereditary haemochromatosis was based on the presence of bioptically confirmed excessive parenchymal iron accumulation without any indication of secondary iron overload, the increased percentage saturation of transferrin, and the increased serum ferritin concentration. In accordance with the recessive mode of inheritance, and maybe the sex difference also, his parents as well as his two sisters and half-brother had no symptoms of hereditary haemochromatosis. The currently applied definition of hereditary haemochromatosis also requires homozygosity for the haemochromatosis alleles. ${ }^{2}$ Our patient is heterozygous for the HLA-A and B haplotypes, but presumably homozygous for the haemochromatosis allele, which, owing to family relations is not accessible for investigation. The two most common HLA alleles associated with hereditary haemochromatosis in Denmark are A3 and B7, alleles which were both found in our patient. HLA-A3 occurred in about $75 \%$ of patients with hereditary haemochromatosis compared with $28-30 \%$ in controls in Europe, the United States, and Australia. ${ }^{5}$ Usually those who are heterozygous for the haemochromatosis allele do not present with clinical disease or develop massive body iron overload, but a proportion may display biochemical abnormalities (most commonly increased transferrin saturation) ${ }^{6}$

Excessive metal accumulation in the CNS is known to cause neurological symptoms. Wilson's disease (hepatolenticular degeneration) is an autosomal recessive condition, located on chromosome $13 \mathrm{q}$ with abnormal copper deposition in the brain (particularly the basal ganglia), liver, cornea, and other organs. Usually the neurological onset is in adolescence, with symptoms such as clumsiness, personality change, reduced mental performance, tremor, rigidity, bradykinesia, dystonia, chorea, incoordination, ataxia, dysathria and, rarely, seizures. ${ }^{7}$ Almost always a Kayser-Fleischer ring at the edge of the cornea is seen. Confirmation of the diagnosis by DNA analysis is possible. Biochemically the diagnosis is confirmed by a low plasma ceruloplasmin, a low serum copper, a high urinary copper excretion, and a high concentration of copper in the liver. Thus this condition was ruled out in our patient.
Locally increased iron concentration in the basal ganglia, not related to any abnormality in the general iron metabolism, with axonal swellings and neuronal degeneration, is pathognomonic for the Hallervorden-Spatz syndrome; characterised by onset in childhood, progressive dementia, bradykinesia, rigidity, spasticity, dystonia, and involuntary movements. ${ }^{8}$ The inheritance is recessive, and a genetic localisation is not established. Eidelberg et al reported on three adult onset single cases, all mentally retarded women, in whom symptoms started between 31 and 42 years of age. ${ }^{9}$ Three sibs, with late age of onset from early adolescence to 55 years of age, with Hallervorden-Spatz disease presenting as familial parkinsonism, were described by Jankovic et $a l^{10} \mathrm{~A}$ biochemical analysis of the brain of the oldest man affected showed considerable loss of dopamine in the nigrostriatal areas, a feature which may explain the beneficial effect of levodopa seen in our patient.

Miyajima et al described a 52 year old woman with familial hypoceruloplasminaemia, blepharospasm, and retinal degeneration. This disorder is dominantly inherited, located on chromosome 3q, and caused by apoceruloplasmin deficiency, which is thought to be causally linked to the iron deposition in the basal ganglia and other organs, shown by kinetics, CT, and histochemical studies. ${ }^{11}$

Parkinson-like syndromes have also been reported after chronic manganese and thallium intoxication, but never in association with hereditary haemochromatosis. Dementia, ataxia, rigidity, and myoclonic jerks were described in two patients with idiopathic haemochromatosis. ${ }^{12}$ Both exhibited slight abnormalities of liver function. Patient 1 had normal brain CT. A postmortem micronodular cirrhosis was found, but no specific changes were noted on neuropathological investigation. A small amount of iron in astrocytes and in the basal ganglia was not thought to represent a pathognomonic change. Patient 2 had a history of alcohol abuse. Cerebrum CT and postmortem examination were not performed. The authors concluded that cirrhosis of the liver, although clinically occult, were the cause of the syndrome of chronic hepatocerebral degeneration.

Furthermore, it has been proposed that a selective increase of iron in the substantia nigra might be linked to the neurodegenerative aspects of Parkinson's disease, involving iron and iron-melanin induced membrane lipid peroxidation, ${ }^{13}$ but this issue still remains to be solved.

Brain MRI showed pronounced signal decrease in the caudate nucleus, the inner segment of the globus pallidus, the red nucleus, substantia nigra, and the dentate nucleus. Several MR studies performed in extrapyramidal movement disorders including Parkinson's disease, multiple system atrophy, Huntingtons disease, and Hallervorden-Spatz disease have shown a close correlation 
between brain iron concentration and $\mathrm{T} 2$ relaxation time within these regions. ${ }^{14} 15$

A recent study by Chen et al on postmortem brain tissue from patients with Parkinson's disease and Huntington's disease showed a trend towards T2 shortening with increasing iron concentration within both the putamen and the globus pallidus in Parkinson's disease and within the globus pallidus in Huntington's disease. In Huntington's disease the highest iron concentration and the longest T2 values were in the putamen, and it was concluded that signal intensity of the basal ganglia on T2 weighted images may be determined by several factors; however, the fact that iron deposition causes a reduction in T2 relaxation was not disputed. ${ }^{16}$

In the present patient extremely low signal intensity was found in the liver on gradient echo MRI consistent with the finding of bioptically confirmed excessive iron accumulation in the liver. A strong correlation has previously been shown between signal intensity and iron concentration in the liver. ${ }^{17}$ This finding and that of reduced signal intensity within the mentioned brain areas in our patient suggests that increased iron accumulation may take place in the brain of patients with hereditary haemochromatosis.

In conclusion, it is therefore likely that excessive iron deposition in the basal ganglia and cerebellum, as shown on MRI, is causally linked to the parkinsonian syndrome in our patient. Although he has presently not improved neurologically on the phlebotomy treatment there is still a possibility that he may do so, because serum ferritin is not yet within the normal range. Hereditary haemochromatosis is not usually considered in the differential diagnosis of parkinsonian syndromes, but because complications of iron induced organ injury may be prevented by phlebotomy, we draw attention to hereditary haemochromatosis as a possible explanation when facing an otherwise "idiopathic" extrapyramidal syndrome.
We thank Dr Lennart Gram and Dr Sven Asger Sørensen who critically reviewed the manuscript. We are grateful to $D$ Margrethe Herning who placed the MRI at our disposal.

1 Stremmel W, Riedel HD, Niederau C, Strohmeyer G. Pathogenesis of genetic haemochromatosis. $\mathcal{f}$ Clin Invest 1993;23:321-29.

2 Edwards CQ. Hemochromatosis and other iron storage disorders. In: Lee GR, Bithell TC, Foerster J, Athers JW, Lukens JN, eds. Wintrobe's Clinical hematology. 9th ed. Philadelphia: Lea and Febiger 1993;872-84.

3 Milder MS, Cook JD, Stray S, Finch CA. Idiopathic hemochromatosis, an interim report. Medicine 1980 59:34-49.

4 Milman N, Graudal N, Nielsen LS, Fenger K. An HLA study in 74 Danish haemochromatosis patients and 21 of their families. Clin Genet 1992;41:6-11.

5 Simon M, Le Mignon L, Fauchet R, et al. A study of 609 HLA haplotypes marking the hemochromatosis gene: (1) mapping of the gene near the HLA-A locus and characters required to define a heterozygous population characters required to define a heterozygous population
and (2) hypothesis concerning the underlying cause of and (2) hypothesis concerning the underlying cause of 1987;41:89-105.

6 Edwards CQ, Dadone M, Skolnick MH, Kushner JP. Hereditary haemochromatosis. Clin Haematol 1982;11: 411-35.

7 Scheinberg IH, Sternlieb I. Wilson's disease. In: Smith $\mathrm{LH} \mathrm{Jr}$, ed. Major problems in internal medicine. Vol XXIII. Philadelphia: WB Saunders Co, 1984.

8 Hallervorden J, Spatz H. Eigenartige Erkrankung im extrapyramidalen System mit besonderer Beteiligung des Globus pallidus und der Substantia nigra. $Z$ Neurol Psychiat 1922;79:254-302.

9 Eidelberg D, Sotrel A, Joachim C, et al. Adult onset Hallervorden-Spatz disease with neurofibrillary patholHallervorden-Spatz disease with neurofibrillary pathol110:993-1013.

10 Jankovic J, Kirkpatrick JB, Blomquist KA, Langlais PJ, Bird ED. Late-onset Hallervorden-Spatz disease presenting as familial parkinsonism. Neurology 1985;35: 227-34

11 Miyajima H, Nishimura Y, Mizoguchi K, Sakamoto M Shimizu T, Honda N. Familial apoceruloplasmin deficiency associated with blepharospasm and retinal degeneration. Neurology 1987;37:761-7.

12 Jones HR Jr, Hedley-Whyte ET. Idiopathic hemochromatosis (IHC): dementia and ataxia as presenting signs. Neurology 1983;33:1479-83.

13 Youdim MBH, Ben-Shachar D, Riederer P. Is Parkinson's disease a progressive siderosis of substantia nigra resulting in iron and melanin induced neuronigra resulting in iron and melanin induced neuration? Acta Neurol Scand 1989;126:47-54.

14 Drayer BP. Magnetic resonance imaging and extrapyramidal movement disorders. Eur Neurol 1989;29(suppl 1):9-12.

15 Savoiardo M, Halliday WC, Nardocci N, et al. Hallervorden-Spatz disease: MR and pathologic findings. AFNR Am $\mathcal{F}$ Neuroradiol 1993;14:155-62.

16 Chen JC, Hardy PA, Kucharczyk W, et al. MR of human postmortem brain tissue: correlative study between $\mathrm{T} 2$ and assays of iron and ferritin in Parkinson's and Huntington's disease. AfNR Am $\mathcal{F}$ Neuroradiol 1993;14 275-81.

17 Thomsen $\mathrm{C}$, Wiggers $\mathrm{P}$, Ring-Larsen $\mathrm{H}$, et al. Identification of patients with hereditary haemochromatosis by magnetic resonance imaging and spectromatosis by magnetic resonance imaging and spectroImaging 1992;10:867-79. 